N. Doi

Nagoya Math. J.

Vol. 78 (1980), 177-188

\title{
ON COMPACT MINIMAL HYPERSURFACES IN A SPHERE WITH CONSTANT SCALAR CURVATURE
}

\author{
NAOYA DOI
}

\section{§1. Introduction}

Let $M$ be an $n$-dimensional hypersurface immersed in the $(n+1)$ dimensional unit sphere $S^{n+1}$ with the standard metric by an immersion $f$. We denote by $A$ the second fundamental form of the immersion $f$ which is considered as a symmetric linear transformation of each tangent space $T_{x} M$, i.e. for an arbitrary point $x$ of $M$ and the unit normal vector field $\xi$ defined in a neighborhood of $x, A$ is given by $f_{*}(A X)=-\bar{D}_{f_{*} X} \xi$ where $\bar{\nabla}$ is the covariant differentiation in $S^{n+1}$ and $X \in T_{x} M$. Thus, $A$ depends on the orientation of the unit normal vector field $\xi$ and, in general, it is locally defined on $M . \quad M$ together with the immersion $f$ is said to be minimal when the mean curvature $\operatorname{tr} A / n$ identically vanishes on $M$. Throughout this paper, we assume that $M$ is a compact minimal hypersurface immersed in $S^{n+1}$ and the square of the length $|A|^{2}$ of the second fundamental form is constant. The assumption that $|A|^{2}$ is constant is equivalent to the one that the scalar curvature $s$ of $M$ is constant, since $s$ is given by $s=n(n-1)-|A|^{2}$ from the well-known Gauss equation.

In [8], Simons proved that $\int_{M}\left(n-|A|^{2}\right)|A|^{2} d V \leqq 0$ for compact minimal hypersurfaces in $S^{n+1}$ and the equality holds if and only if $A$ is covariant constant, i.e. $\nabla A \equiv 0$. The study on the case $\nabla A \equiv 0$ was done by Chern-do Carmo-Kobayashi [2] and Lawson [4].

To investigate the case $|A|^{2}>n$, we must consider the situation $\nabla A \neq 0$. The purpose of this paper is to give an integral inequality (Theorem 1) and its applications. This inequality seems to be more delicate than Simons' one in the sense that the equality in it does not necessarily imply $\nabla A \equiv 0$. In section 4 , we consider a 3-dimensional minimal hypersurface $M^{3}$ in $S^{4}$ and we characterize the cases that $M^{3}$ has positive semi-definite Ricci tensor (Corollary 1 ) and $M^{3}$ has sectional

Received February 19, 1979. 
curvature $\leqq 1$ (Theorem 2). These are the partial answers for the problem proposed by Chern-do Carmo-Kobayashi [2] that for minimal hypersurfaces in $S^{n+1}$ with constant $|A|^{2}$, what is the possible next value for $|A|^{2}$ greater than $n$.

\section{§2. Preliminaries}

Let $M$ be an $n$-dimensional compact oriented Riemannian manifold with the Riemannian metric $\langle$,$\rangle and the covariant differentiation \nabla$. All tensors on $M$ have a natural inner product and $\nabla_{X}$ extends as a derivation to all tensors. We also denote them by $\langle$,$\rangle and \nabla_{X}$. Let $E$ be a Riemannian vector bundle over $M$ with the inner product (, ) of each fibre $E_{x}$ and the metric connection $\tilde{\nabla}$ corresponding to the inner product (,). For any cross-section $\eta$ of $E$ and any tangent vectors $X, Y \in T_{x} M$ at each point $x$ of $M$, we define

$$
\tilde{\nabla}_{X, Y} \eta=\tilde{\nabla}_{X} \tilde{\nabla}_{\bar{Y}} \eta-\tilde{\nabla}_{\nabla_{X} \bar{Y}} \eta \in E_{x}
$$

where $\bar{Y}$ is a local extention of $Y$ to a vector field on $M$. It can be easily seen that this definition does not depend on the choice of $\bar{Y}$.

Let $\phi$ be a symmetric bilinear form on $M$. In [1], Cheng-Yau introduced a differential operator $\square$ on functions on $M$ which is associated to $\phi$. We can easily see that this operator is naturally extended to the one on cross-sections of any Riemannian vector bundle over $M$ :

Definition. For any cross-section $\eta$ of $E$, we define

$$
\square \eta=\sum_{i, j=1}^{n} \phi\left(e_{i}, e_{j}\right) \tilde{V}_{e_{i}, e_{j}} \eta
$$

where $e_{1}, \cdots, e_{n}$ denotes an orthonormal frame of each tangent space $T_{x} M$. This definition does not depend on the choice of the orthonormal frame and coincides with the one given in [1] when $\eta$ is a function on $M$. Note that when $\phi\left(e_{i}, e_{j}\right)=\delta_{i j}, \square$ is the Laplace operator $\Delta$ (c.f. Simons [8] and Nomizu-Smyth [5]).

Proposition 1. If $\phi$ satisfies $\sum_{i=1}^{n}\left(\nabla_{e_{i}} \phi\right)\left(X, e_{i}\right)=0$ for any tangent vector $X \in T_{x} M$ at each point $x$ of $M$, then for any cross-sections $\eta, \nu$ of $E$ we have 


$$
\int_{M}(\square \eta, \nu) d V=-\int_{M}\left\{\sum_{i, j=1}^{n} \phi\left(e_{i}, e_{j}\right)\left(\tilde{\nabla}_{e_{i}} \eta, \tilde{\nabla}_{e_{j}} \nu\right)\right\} d V
$$

where $d V$ is the volume form of $M$. Thus, $\square$ is self-adjoint.

Proof. Let $E_{1}, \cdots, E_{n}$ be a local orthonormal frame field on $M$ and $\Omega$ be the $(n-1)$-form on $M$ defined by

$$
\left.\Omega=\sum_{i, j=1}^{n} \phi\left(E_{i}, E_{j}\right)\left(\tilde{V}_{E_{j}} \eta, \nu\right) E_{i}\right\rfloor d V .
$$

Then, by a straightforward calculation we have

$$
d \Omega=\sum_{i, j=1}^{n} \phi\left(E_{i}, E_{j}\right)\left(\tilde{\nabla}_{E_{i}} \eta, \tilde{\nabla}_{E_{j}} \nu\right) d V+\sum_{i, j=1}^{n} \phi\left(E_{i}, E_{j}\right)\left(\tilde{\nabla}_{E_{i}, E_{j}} \eta, \nu\right) d V
$$

if $\sum_{i=1}^{n}\left(\nabla_{E_{i}} \phi\right)\left(X, E_{i}\right)=0$ for any tangent vector $X$. This together with the Stokes' Theorem implies (2.1).

Now, in the following, we always assume that $M$ is isometrically immersed in the $(n+1)$-dimensional unit sphere $S^{n+1}$ as a minimal hypersurface. Let $A$ be the second fundamental form of this immersion as in the introduction. Let $R$ be the curvature tensor of $M$. Then the Gauss equation and the Codazzi equation are respectively expressed as

$$
\begin{aligned}
\langle R(X, Y) Z, W\rangle= & \langle X, W\rangle\langle Y, Z\rangle-\langle X, Z\rangle\langle Y, W\rangle \\
& +\langle A X, W\rangle\langle A Y, Z\rangle-\langle A X, Z\rangle\langle A Y, W\rangle
\end{aligned}
$$

and

$$
\left(\nabla_{X} A\right) Y=\left(\nabla_{Y} A\right) X
$$

for any tangent vectors $X, Y, Z, W \in T_{x} M$ at each point $x$ of $M$. By means of (2.2), the Ricci tensor Ric of $M$ is expressed as

$$
\operatorname{Ric}(X, Y)=n-1-\left\langle A^{2} X, Y\right\rangle
$$

for $X, Y \in T_{x} M$.

\section{§3. The integral inequality}

Let $\phi$ be a symmetric bilinear form locally defined on $M$ by $\phi(X, Y)$ $=\langle A X, Y\rangle$ for any tangent vectors $X, Y$. Let $\square_{\phi}$ be the operator defined in section 2 which is associated to $\phi$. Clearly $\square_{\phi}$ is locally defined, however, $\square_{\phi} A$ is globally defined on $M$, since it does not depend on the. 
orientation of the unit normal vector field. Since $A^{2}$ is globally defined, we define a symmetric bilinear form $\psi$ on $M$ by $\psi(X, Y)=\left\langle A^{2} X, Y\right\rangle$ for any tangent vectors $X, Y$ and denote its associated operator by $\square_{\psi}$. From the Codazzi equation and the assumption that $\operatorname{tr} A=0$ and $|A|^{2}$ is constant, we can easily see that $\phi$ and $\psi$ satisfy the assumption in Proposition 1.

Lemma 1. Let $M$ be a minimal hypersurface in $S^{n+1}$. For any tangent vectors $X, Y \in T_{x} M$ at each point $x$ of $M$, we have

$$
\begin{aligned}
\left\langle\left(\square_{\phi} A\right) X, Y\right\rangle= & |A|^{2}\left\langle A^{2} X, Y\right\rangle-\operatorname{tr} A^{3}\langle A X, Y\rangle-|A|^{2}\langle X, Y\rangle \\
& +\left\langle\nabla_{X, Y} A, A\right\rangle .
\end{aligned}
$$

Proof. Choose the orthonormal frame $e_{1}, \cdots, e_{n}$ of $T_{x} M$ such that $A e_{i}=\lambda_{i} e_{i}$ for all $i$. Let $\bar{X}, \bar{Y}$ and $E_{1}, \cdots, E_{n}$ be the local extensions of $X, Y$ and $e_{1}, \cdots, e_{n}$ respectively to the vector fields on $M$ such that $\nabla_{e_{i}} \bar{X}$ $=0, \nabla_{e_{i}} \bar{Y}=0$ and $\nabla_{e_{i}} E_{j}=0$ for all $i, j$. Using the Codazzi equation, we have

$$
\begin{aligned}
\left\langle\left(\square_{\phi} A\right) X, Y\right\rangle & =\sum_{i, j=1}^{n} \phi\left(e_{i}, e_{j}\right)\left\langle\nabla_{e_{i}, e_{j}} A X, Y\right\rangle \\
& =\sum_{i=1}^{n} \lambda_{i}\left\langle\nabla_{e_{i}} \nabla_{E_{i}} A X, Y\right\rangle=\sum_{i=1}^{n} \lambda_{i}\left\langle\nabla_{e_{i}}\left(\nabla_{E_{i}} A \bar{X}\right), Y\right\rangle \\
& =\sum_{i=1}^{n} \lambda_{i}\left\langle\nabla_{e_{i}}\left(\nabla_{X} A E_{i}\right), Y\right\rangle=\sum_{i=1}^{n} \lambda_{i}\left\langle\nabla_{e_{i}} \nabla_{X} A e_{i}, Y\right\rangle .
\end{aligned}
$$

Extending the curvature operator $R\left(e_{i}, X\right)$ as a derivation to all tensor fields on $M$, we have

$$
\begin{aligned}
\sum_{i=1}^{n} \lambda_{i}\left\langle\nabla_{e_{i}} \nabla_{X} A e_{i}, Y\right\rangle= & \sum_{i=1}^{n} \lambda_{i}\left\langle\left(R\left(e_{i}, X\right) A\right) e_{i}, Y\right\rangle \\
& +\sum_{i=1}^{n} \lambda_{i}\left\langle\nabla_{X} \nabla_{E_{i}} A e_{i}, Y\right\rangle .
\end{aligned}
$$

Since $\left(R\left(e_{i}, X\right) A\right) e_{i}=R\left(e_{i}, X\right)\left(A e_{i}\right)-A\left(R\left(e_{i}, X\right) e_{i}\right)$, by means of the Gauss equation (2.2), a straightforward calculation shows that

$$
\sum_{i=1}^{n} \lambda_{i}\left\langle\left(R\left(e_{i}, X\right) A\right) e_{i}, Y\right\rangle=|A|^{2}\left\langle A^{2} X, Y\right\rangle-\operatorname{tr} A^{3}\langle A X, Y\rangle-|A|^{2}\langle X, Y\rangle .
$$

From the symmetry property of $A$ and the Codazzi equation, we have

$$
\sum_{i=1}^{n} \lambda_{i}\left\langle\nabla_{X} \nabla_{E_{i}} A e_{i}, Y\right\rangle=\sum_{i=1}^{n} \lambda_{i} \nabla_{X}\left\langle\left(\nabla_{E_{i}} A\right) E_{i}, \bar{Y}\right\rangle
$$




$$
\begin{aligned}
& =\sum_{i=1}^{n} \lambda_{i} \nabla_{X}\left\langle\left(\nabla_{\bar{Y}} A\right) E_{i}, E_{i}\right\rangle=\sum_{i=1}^{n} \lambda_{i}\left\langle\nabla_{X} \nabla_{\bar{Y}} A e_{i}, e_{i}\right\rangle \\
& =\sum_{i=1}^{n}\left\langle\nabla_{X, Y} A e_{i}, A e_{i}\right\rangle=\left\langle\nabla_{X, Y} A, A\right\rangle .
\end{aligned}
$$

From (3.1), (3.2), (3.3) and (3.4), we obtain Lemma 1.

q.e.d.

THEOREM 1. Let $M$ be a compact oriented minimal hypersurface immersed in $S^{n+1}$. If the scalar curvature of $M$ is constant, then we have

$$
\int_{M}\left\{\left(|A|^{2}-4 n\right) \operatorname{tr} A^{4}+3\left(\operatorname{tr} A^{3}\right)^{2}+3|A|^{4}\right\} d V \geqq 0 .
$$

The equality in (3.5) holds if and only if

$$
\sum_{i=1}^{n}\left\{\left\langle\left(\nabla_{e_{i}} A\right)^{2}, A^{2}\right\rangle+2\left\langle\left(\nabla_{e_{i}} A\right) \cdot A, A \cdot\left(\nabla_{e_{i}} A\right)\right\rangle\right\}=0
$$

where $e_{1}, \cdots, e_{n}$ is an orthonormal frame of each tangent space $T_{x} M$.

Proof. The assumption that the scalar curvature is constant implies that $|A|^{2}$ is constant and $\psi$ satisfies the assumption in Proposition 1.

From Lemma 1 and the definition of $\psi$, we see that

$$
\left\langle\square_{\phi} A, A^{2}\right\rangle-\left\langle\square_{\psi} A, A\right\rangle=|A|^{2} \operatorname{tr} A^{4}-\left(\operatorname{tr} A^{3}\right)^{2}-|A|^{4} .
$$

Thus, from (2.1) we have

$$
\begin{aligned}
\int_{M}\left\{|A|^{2}\right. & \left.\operatorname{tr} A^{4}-\left(\operatorname{tr} A^{3}\right)^{2}-|A|^{4}\right\} d V \\
= & \int_{M}\left\{\left\langle\square_{\phi} A, A^{2}\right\rangle-\left\langle\square_{\psi} A, A\right\rangle\right\} d V \\
= & -\int_{M}\left\{\sum_{i=1}^{n} \lambda_{i}\left\langle\nabla_{e_{t}} A, \nabla_{e_{t}} A^{2}\right\rangle\right\} d V+\int_{M}\left\{\sum_{i=1}^{n} \lambda_{i}^{2}\left\langle\nabla_{e_{i}} A, \nabla_{e_{i}} A\right\rangle\right\} d V
\end{aligned}
$$

where $e_{1}, \cdots, e_{n}$ is the orthonormal frame of each tangent space $T_{x} M$ such that $A e_{i}=\lambda_{i} e_{i}$ for all $i$. Now, we shall denote $\left\langle\left(\nabla_{e_{i}} A\right) e_{j}, e_{k}\right\rangle=h_{i j k}$. Then, from the Codazzi equation, we can see that

$$
\begin{aligned}
\sum_{i=1}^{n} \lambda_{i}\left\langle\nabla_{e_{i}} A, \nabla_{e_{i}} A^{2}\right\rangle \\
\quad=2 \sum_{i=1}^{n}\left\langle\left(\nabla_{e_{i}} A\right) \cdot A, A \cdot\left(\nabla_{e_{i}} A\right)\right\rangle=2 \sum_{i, j, k=1}^{n} \lambda_{i} \lambda_{j}\left(h_{i j k}\right)^{2}
\end{aligned}
$$


and

$$
\sum_{i=1}^{n} \lambda_{i}^{2}\left\langle\nabla_{e_{i}} A, \nabla_{e_{i}} A\right\rangle=\sum_{i=1}^{n}\left\langle\left(\nabla_{e_{i}} A\right)^{2}, A^{2}\right\rangle=\sum_{i, j, k=1}^{n} \lambda_{i}^{2}\left(h_{i j k}\right)^{2}
$$

For the sake of simplicity, we denote (3.7) by $S$ and (3.8) by $T$. Then, (3.6) is expressed as

$$
\int_{M}\left\{|A|^{2} \operatorname{tr} A^{4}-\left(\operatorname{tr} A^{3}\right)^{2}-|A|^{4}\right\} d V=\int_{M}(T-S) d V .
$$

On the other hand, by the result in Simons [8] that $\Delta A=\left(n-|A|^{2}\right) A$ for any minimal hypersurface in $S^{n+1}$, we can obtain another relation between $T$ and $S$, that is

$$
\int_{M}\left(|A|^{2}-n\right) \operatorname{tr} A^{4} d V=\int_{M}\left(2 T+\frac{1}{2} S\right) d V
$$

since $\left\langle\Delta A, A^{3}\right\rangle=\left(n-|A|^{2}\right) \operatorname{tr} A^{4},\left\langle\nabla A, \nabla A^{3}\right\rangle=2 T+\frac{1}{2} S$ and

$$
\int_{M}\left\langle\Delta A, A^{3}\right\rangle d V=-\int_{M}\left\langle\nabla A, \nabla A^{3}\right\rangle d V
$$

Compairing (3.9) with (3.10), we have

$$
\int_{M}\left\{\left(|A|^{2}-4 n\right) \operatorname{tr} A^{4}+3\left(\operatorname{tr} A^{3}\right)^{2}+3|A|^{4}\right\} d V=\int_{M} 5(T+S) d V .
$$

Noting that the indices $i, j, k$ in $h_{i j k}$ can be mutually commuted, from (3.7) and (3.8), we see

$$
S=2 \sum_{i, j, k=1}^{n} \lambda_{i} \lambda_{j}\left(h_{i j k}\right)^{2}=\frac{2}{3} \sum_{i, j, k=1}^{n}\left(\lambda_{i} \lambda_{j}+\lambda_{j} \lambda_{k}+\lambda_{k} \lambda_{i}\right)\left(h_{i j k}\right)^{2}
$$

and

$$
T=\sum_{i, j, k=1}^{n} \lambda_{i}^{2}\left(h_{i j k}\right)^{2}=\frac{1}{3} \sum_{i, j, k=1}^{n}\left(\lambda_{i}^{2}+\lambda_{j}^{2}+\lambda_{k}^{2}\right)\left(h_{i j k}\right)^{2} .
$$

Thus, we have

$$
T+S=\frac{1}{3} \sum_{i, j, k=1}^{n}\left(\lambda_{i}+\lambda_{j}+\lambda_{k}\right)^{2}\left(h_{i j k}\right)^{2} \geqq 0 .
$$

This together with (3.11) implies (3.5) in Theorem 1. q.e.d.

Remark 1. The symmetric bilinear form defined by $\psi^{\prime}\left(e_{i}, e_{j}\right)=$ 
$\left\langle A^{2} e_{i}, e_{j}\right\rangle-\frac{|A|^{2}}{2} \delta_{i j}$ satisfies the assumption in Proposition 1 without assuming that $|A|^{2}$ is constant. Using $\psi^{\prime}$, by a similar calculation to the one in the proof of Lemma 1, we can see that (3.9) is replaced by

$$
\int_{M}\left\{|A|^{2} \operatorname{tr} A^{4}-\left(\operatorname{tr} A^{3}\right)^{2}-|A|^{4}+\frac{1}{4}|A|^{2} \Delta|A|^{2}\right\} d V=\int_{M}(T-S) d V .
$$

Remark 2. Clearly, if $\nabla A \equiv 0, T+S \equiv 0$ and the equality in (3.5) holds. The following example, which is one of the homogeneous minimal hypersurfaces in a sphere demonstrated by Hsiang [3], asserts that $T+S$ $\equiv 0$ does not always imply $\nabla A \equiv 0$.

Let $\subseteq$ be the set of $3 \times 3$ symmetric real matrices with trace 0 . $\subseteq$ is considered as a 5-dimensional vector space. We define the inner product in $\widetilde{S}$ by $(B, C)=\operatorname{tr} B C$ for $B, C \in \widetilde{S}$. Let $\mathbb{S}^{\prime}=\{B \in \mathfrak{S} ;(B, B)=1\}$. $\widetilde{S}^{\prime}$ is identified with the 4-dimensional unit sphere $S^{4}$ in $R^{5}$. Let $S O(3)$ act on $\widetilde{S}^{\prime}$ by conjugation. We define the map $\tau$ of $S O(3)$ into $S^{4}$ by

$$
\tau: S O(3) \rightarrow \mathbb{S}^{\prime} \cong S^{4}, \quad \tau(g)=g B_{0} g^{-1}
$$

where

$$
B_{0}=\left(\begin{array}{ccc}
1 / \sqrt{2} & 0 & 0 \\
0 & -1 / \sqrt{2} & 0 \\
0 & 0 & 0
\end{array}\right) \in \mathbb{S}^{\prime}
$$

By a direct computation, we see that

(1) $\tau$ is an immersion,

(2) The induced metric on $S O(3)$ by $\tau$ is left invariant, and with respect to it, the left invariant vector fields

$$
X_{1}=\left(\begin{array}{rrr}
0 & 0 & 1 \\
0 & 0 & 0 \\
-1 & 0 & 0
\end{array}\right), \quad X_{2}=\left(\begin{array}{rrr}
0 & 0 & 0 \\
0 & 0 & 1 \\
0 & -1 & 0
\end{array}\right), \quad X_{3}=\left(\begin{array}{ccc}
0 & 1 / 2 & 0 \\
-1 / 2 & 0 & 0 \\
0 & 0 & 0
\end{array}\right) \in \mathfrak{g n}(3)
$$

are the orthonormal frame field on $S O(3)$,

(3) The second fundamental form $A$ of $\tau$ is given by

$$
A\left(X_{1}\right)=\sqrt{3} X_{1}, \quad A\left(X_{2}\right)=-\sqrt{3} X_{2}, \quad A\left(X_{3}\right)=0
$$

for the unit normal vector with suitable orientation at each point of $M$, and hence $\tau$ is the minimal immersion. In this case, the left hand side 
of (3.5) vanishes, and so $T+S=0$. Since $|A|^{2}=6$, by the result in Simons [8] that $\Delta A=\left(3-|A|^{2}\right) A$, we have

$$
\left(3-|A|^{2}\right)|A|^{2}=\langle\Delta A, A\rangle=-\langle\nabla A, \nabla A\rangle+\frac{1}{2} \Delta|A|^{2}=-\langle\nabla A, \nabla A\rangle,
$$

and hence $\langle\nabla A, \nabla A\rangle=18$. This implies $\nabla A \neq 0$.

\section{§4. Applications}

In this section, we mainly study 3-dimensional minimal hypersurfaces in $S^{4}$ with constant scalar curvature.

The Clifford hypersurfaces $M_{p, n-p} p=1, \cdots, n-1$ in $S^{n+1}$ are defined by

$$
M_{p, n-p}=\left\{(x, y) \in \boldsymbol{R}^{p+1} \times \boldsymbol{R}^{n-p+1} \cong \boldsymbol{R}^{n+2} ;|x|^{2}=\frac{p}{n},|y|^{2}=\frac{n-p}{n}\right\}
$$

It is proved by Chern-do Carmo-Kobayashi [2] and Lawson [4] that a great sphere and the Clifford hypersurfaces are the only compact minimal hypersurfaces in $S^{n+1}$ satisfying $|A|^{2} \leqq n$. They are also the only compact minimal hypersurfaces in $S^{n+1}$ which have non-negative sectional curvature, since from this condition together with the fact $\operatorname{tr} A=0$, it directly follows that $|A|^{2} \leqq n$. This result is given in Nomizu-Smyth [5].

For compact minimal hypersurfaces in $S^{4}$, we show that a great sphere and the Clifford hypersurfaces $M_{1,2}$ can be characterized by the weaker condition than sectional curvature's one under the assumption that $|A|^{2}$ is constant.

CoRollary 1. Let $M$ be a compact minimal hypersurface in $S^{4}$ with constant scalar curvature. If $M$ has positive semi-definite Ricci tensor, $M$ is realized only by a great sphere or the Clifford hypersurfaces $M_{1,2}$.

Proof. Let $e_{1}, e_{2}, e_{3}$ be the orthonormal frame of each tangent space $T_{x} M$ such that $A e_{i}=\lambda_{i} e_{i} i=1,2,3$. Since $\operatorname{tr} A=0$, it can be easily seen that $\operatorname{tr} A^{3}=3 \operatorname{det} A$ and $\operatorname{tr} A^{4}=\frac{1}{2}|A|^{4}$. Thus, (3.5) in Theorem 1 takes the form

$$
\left.\int_{M} \frac{1}{2}|A|^{4}\left(|A|^{2}-6\right)+27(\operatorname{det} A)^{2}\right\} d V \geqq 0 .
$$

From (2.4), the condition that the Ricci tensor is positive semi-definite 
implies $2-\lambda_{i}^{2} \geqq 0 i=1,2,3$. Hence,

$$
\begin{aligned}
0 \geqq \prod_{i=1}^{3}\left(\lambda_{i}^{2}-2\right) & =\lambda_{1}^{2} \lambda_{2}^{2} \lambda_{3}^{2}-\left(\sum_{i=1}^{3} \lambda_{i}^{2}\right)^{2}+\sum_{i=1}^{3} \lambda_{i}^{4}+4 \sum_{i=1}^{3} \lambda_{i}^{2}-8 \\
& =(\operatorname{det} A)^{2}-|A|^{4}+\operatorname{tr} A^{4}+4|A|^{2}-8 \\
& =(\operatorname{det} A)^{2}-\frac{1}{2}|A|^{4}+4|A|^{2}-8
\end{aligned}
$$

From this inequality together with (4.1), we have

$$
\int_{M} \frac{1}{2}\left(|A|^{2}-3\right)\left(|A|^{2}-12(\sqrt{2}-1)\right)\left(|A|^{2}+12(\sqrt{2}+1)\right) d V \geqq 0 .
$$

Since $\lambda_{i}^{2} \leqq 2 i=1,2,3$ and $\operatorname{tr} A=0$, we see that $|A|^{2} \leqq 4$. Thus, from the assumption that $|A|^{2}$ is constant and the inequality (4.2), it follows that $|A|^{2} \leqq 3$. This implies the desired result in Corollary 1 . q.e.d.

Now, we shall consider a compact minimal hypersurface $M$ in $S^{n+1}$ of which sectional curvature is bounded above by 1 . Hypersurfaces in a space of constant curvature $c$ which have sectional curvature $\leqq c$ are studied in Pinl-Ziller [6] and Yau [10], and it is easily verified that rank $A$ $\leqq 2$ everywhere. The possible values for $|A|^{2}$ of such hypersurfaces seem to be unknown and the rest of this paper is devoted to studying this problem. In our case, since $\operatorname{tr} A=0$, the fact that $\operatorname{rank} A \leqq 2$ implies $\operatorname{tr} A^{3}=0$ and $\operatorname{tr} A^{4}=\frac{1}{2}|A|^{4}$. Thus, (3.5) in Theorem 1 becomes

$$
\int_{M} \frac{1}{2}|A|^{4}\left(|A|^{2}-2(2 n-3)\right) d V \geqq 0
$$

Hence, we have

Corollary 2. Let $M$ be a compact minimal hypersurface in $S^{n+1}$ with constant scalar curvature. If sectional curvature of $M$ is bounded above by $1, M$ is a great sphere or $|A|^{2} \geqq 2(2 n-3)$, i.e. scalar curvature $s \leqq(n-2)(n-3)$.

As for $n=3$, the lower bound 6 of the above inequality $|A|^{2} \geqq 6$ is actually realized by the immersion $\tau$ given in Remark 2. The following Theorem characterizes this immersion.

Theorem 2. Let $M$ be a compact minimal hypersurface immersed in 
$S^{4}$ with constant scalar curvature. Assume that sectional curvature of $M$ is bounded above by 1 and $|A|^{2} \leqq 6$, then we have $|A|^{2}=0$ or $|A|^{2}=6$. The case $|A|^{2}=0$ is realized only by a great sphere and the case $|A|^{2}=6$ is realized only by the immersion $\tau$ up to the rigid motion of $S^{4}$.

Proof. The first statement that if $|A|^{2} \leqq 6,|A|^{2}=0$ or $|A|^{2}=6$ directly follows from (4.3). The case $|A|^{2}=0$ is trivial. Thus, we shall prove the last statement.

Since $|A|^{2}=6$ and $\operatorname{rank} A \leqq 2, A$ has eigenvalues $\sqrt{3},-\sqrt{3}, 0$ at each point of $M$. Let $E_{1}, E_{2}, E_{3}$ be the local orthonormal frame field defined on sufficiently small open set $U$ in $M$ satisfying

$$
A E_{1}=\sqrt{3} E_{1}, \quad A E_{2}=-\sqrt{3} E_{2}, \quad A E_{3}=0 .
$$

Let $\omega_{1}, \omega_{2}, \omega_{3}$ be the dual frame field of $E_{1}, E_{2}, E_{3}$. We define 1-forms $\omega_{i j}$ $i, j=1,2,3$ by $\omega_{i j}(X)=\left\langle\nabla_{X} E_{i}, E_{j}\right\rangle$ for any tangent vector $X$ of $U$. From the structure equation of $M$, we see

$$
d \omega_{i}=\sum_{j=1}^{3} \omega_{i j} \wedge \omega_{j} \quad \text { for } i=1,2,3 .
$$

Now, since $|A|^{2}=6$, the left hand side of (4.3) vanishes. From Theorem 1 , this implies $T+S=\frac{1}{3} \sum_{i, j, k=1}^{3}\left(\lambda_{i}+\lambda_{j}+\lambda_{k}\right)^{2}\left(h_{i j k}\right)^{2}$ identically vanishes on $M$. In the present case, $h_{i j k}$ is given by $\left\langle\left(\nabla_{E_{i}} A\right) E_{j}, E_{k}\right\rangle$, and setting $\lambda_{1}=\sqrt{3}, \lambda_{2}=-\sqrt{3}, \lambda_{3}=0$, we can easily see that

$$
h_{i i j}=\left\langle\left(\nabla_{E_{i}} A\right) E_{i}, E_{j}\right\rangle=0 \quad \text { for } i, j=1,2,3 \text {. }
$$

Moreover, since $|A|^{2}=6$, from (4.5) and Simons' equation $\Delta A=-3 A$, it follows that

$$
\langle\nabla A, \nabla A\rangle=\frac{1}{2} \Delta|A|^{2}-\langle\Delta A, A\rangle=3|A|^{2}=18
$$

and

$$
\langle\nabla A, \nabla A\rangle=\sum_{i, j, k=1}^{3}\left\langle\left(\nabla_{E_{i}} A\right) E_{j}, E_{k}\right\rangle^{2}=6\left(h_{123}\right)^{2} .
$$

Thus, $\left(h_{123}\right)^{2}$ is always equal to 3 . We may locally assume that

$$
h_{123}=\sqrt{3} \text {. }
$$

Using the fact that $\left\langle A E_{i}, E_{j}\right\rangle=$ constant for all $i, j$, from (4.5) we have 


$$
\begin{aligned}
0 & =\left\langle\left(\nabla_{E_{i}} A\right) E_{i}, E_{j}\right\rangle=E_{i}\left\langle A E_{i}, E_{j}\right\rangle-\left\langle A\left(\nabla_{E_{i}} E_{i}\right), E_{j}\right\rangle-\left\langle A E_{i}, \nabla_{E_{i}} E_{j}\right\rangle \\
& =-\left\langle A E_{j}, \nabla_{E_{i}} E_{i}\right\rangle-\left\langle A E_{i}, \nabla_{E_{i}} E_{j}\right\rangle \\
& =-\lambda_{j} \omega_{i j}\left(E_{i}\right)-\lambda_{i} \omega_{j i}\left(E_{i}\right)=\left(\lambda_{j}-\lambda_{i}\right) \omega_{j i}\left(E_{i}\right)
\end{aligned}
$$

for all $i, j$, where $\lambda_{1}=\sqrt{3}, \lambda_{2}=-\sqrt{3}, \lambda_{3}=0$ and we used the fact $\omega_{i j}$ $=-\omega_{j i}$. From (4.7), we can verify

$$
\omega_{12}=f_{3} \omega_{3}, \quad \omega_{23}=f_{1} \omega_{1}, \quad \omega_{31}=f_{2} \omega_{2}
$$

where $f_{1}, f_{2}, f_{3}$ are functions on $U$. From (4.6), we have

$$
\begin{aligned}
\sqrt{3} & =\left\langle\left(\nabla_{E_{1}} A\right) E_{2}, E_{3}\right\rangle=E_{1}\left\langle A E_{2}, E_{3}\right\rangle-\left\langle A\left(\nabla_{E_{1}} E_{2}\right), E_{3}\right\rangle-\left\langle A E_{2}, \nabla_{E_{1}} E_{3}\right\rangle \\
& =-\lambda_{3} \omega_{23}\left(E_{1}\right)-\lambda_{2} \omega_{32}\left(E_{1}\right)=\sqrt{3} \omega_{32}\left(E_{1}\right) .
\end{aligned}
$$

Thus, $\omega_{32}\left(E_{1}\right)=1$ and $f_{1}=-1$. A similar calculation shows that $\sqrt{3}=$ $h_{312}=2 \sqrt{3} \omega_{12}\left(E_{3}\right)$ and $\sqrt{3}=h_{231}=-\sqrt{3} \omega_{31}\left(E_{2}\right)$. Thus, $f_{2}=-1$ and $f_{3}=$ $\frac{1}{2}$. Hence, (4.4) can be expressed as

$$
d \omega_{1}=\frac{1}{2} \omega_{2} \wedge \omega_{3}, \quad d \omega_{2}=\frac{1}{2} \omega_{3} \wedge \omega_{1}, \quad d \omega_{3}=2 \omega_{1} \wedge \omega_{2} .
$$

Let $X_{1}, X_{2}, X_{3} \in \mathfrak{g}_{0}(3)$ be the left invariant orthonormal frame field on $S O(3)$ as in Remark 2 and $\theta_{1}, \theta_{2}, \theta_{3}$ be its dual frame field. Then the MaurerCartan equation is expressed as

$$
d \theta_{1}=\frac{1}{2} \theta_{2} \wedge \theta_{3}, \quad d \theta_{2}=\frac{1}{2} \theta_{3} \wedge \theta_{1}, \quad d \theta_{3}=2 \theta_{1} \wedge \theta_{2} .
$$

Compairing (4.8) with (4.9), we see that there exists a diffeomorphism $f$ of $U$ into $S O(3)$ for sufficiently small open set $U$ in $M$ and $f$ satisfies $f^{*} \theta_{i}=\omega_{i} i=1,2,3$ (c.f. Șternberg [9]). Since $E_{1}, E_{2}, E_{3}$ and $X_{1}, X_{2}, X_{3}$ are chosen as orthonormal frame fields on $U$ and $S O(3)$ respectively, $f$ is an isometry of $U$ into $S O(3)$, and furthermore $f$ preserves the second fundamental forms of each immersion into $S^{4}$. Therefore, since $M$ is compact, from the fundamental Theorem for hypersurfaces, we can conclude that $M$ coincides with the hypersurface given by the immersion $\tau: S O(3) \rightarrow S^{4}$ up to the rigid motion of $S^{4}$.

q.e.d.

\section{REFERENCES}

[1] S. Y. Cheng and S. T. Yau, Hypersurfaces with constant scalar curvature, Math. Ann. 225 (1977), 195-204. 
[2] S. S. Chern, M. do Carmo and S. Kobayashi, Minimal submanifolds of a sphere with second fundamental form of constant length. Functional Analysis and Related Fields (1970), 59-75.

[ 3 ] W. Y. Hsiang, Remarks on closed minimal submanifolds in the standard $m$-sphere, J. Diff. Geom. 1 (1967), 257-267.

[4] H. B. Lawson, Local rigidity theorems for minimal hypersurfaces, Ann. of Math. 89 (1969), 187-197.

[5] K. Nomizu and B. Smyth, A formula of Simons' type and Hypersurfaces with constant mean curvature, J. Diff. Geom. 3 (1969), 367-377.

[6 ] M. Pinl and W. Ziller, Minimal hypersurfaces in a space of constant curvature, J. Diff. Geom. 11 (1976), 335-343.

[ 7 ] P. J. Ryan, Homogeneity and some curvature conditions for hypersurfaces, Tohoku Math. Journ. 21 (1969), 363-388.

[8] J. Simons, Minimal varieties in riemannian manifolds, Ann. of Math. 88 (1968), $62-105$.

[9] S. Sternberg, Lectures on differential geometry, Prentice-Hall (1964).

[10] S. T. Yau, Submanifolds with constant mean curvature II, Amer. J. of Math. 97 (1973), 76-100.

Department of Mathematics

Nagoya University 